\title{
The hydrogen storage capacity of metal-containing polyacry- lonitrile-based electrospun carbon nanofibers
}

\author{
Byong Chol Bai ${ }^{1,2}$, Jong Gu Kim², Mehraj-ud-din Naik², Ji Sun Im $^{2}$ and Young-Seak Lee, ${ }^{1,2,}$ \\ ${ }^{1}$ Department of Green Energy Technology, Chungnam National University, Daejeon 305-764, Korea \\ ${ }^{2}$ Department of Fine Chemical Engineering and Applied Chemistry, BK21-E2M, Chungnam National University, Daejeon 305-764, \\ Korea
}

\section{Article Info}

Received 9 August 2011

Accepted 7 September 2011

*Corresponding Author

E-mail: youngslee@cnu.ac.kr

Tel: $+82-42-821-7007$

\section{Open Access}

DOI: http://carbonlett.org/

10.5714/CL.2011.12.3.171

This is an Open Access article distributed under the terms of the Creative Commons Attribution Non-Commercial License (http://creativecommons.org/licenses/ by-nc/3.0/) which permits unrestricted non-commercial use, distribution, and reproduction in any medium, provided the original work is properly cited.

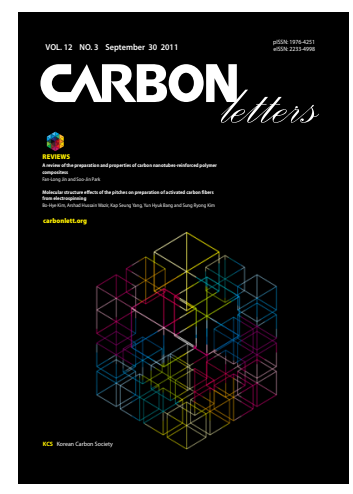

http://carbonlett.org

pISSN: $1976-4251$

elSSN: 2233-4998

Copyright $\odot$ Korean Carbon Society

\begin{abstract}
Polyacrylonitrile-based carbon nanofibers (CNFs) containing Ti and Mn were prepared by electrospinning. The effect of metal content on the hydrogen storage capacity of the nanofibers was evaluated. The nanofibers containing $\mathrm{Ti}$ and $\mathrm{Mn}$ exhibited maximum hydrogen adsorption capacities of 1.6 and $1.1 \mathrm{wt} \%$, respectively, at $303 \mathrm{~K}$ and $9 \mathrm{MPa}$. Toward the development of an improved hydrogen storage system, the optimum conditions for the production of metalized CNFs were investigated by characterizing the specific surface areas, pore volumes, sizes, and shapes of the fibers. According to the results of Brunauer-EmmettTeller analysis, the activation of the CNFs using potassium hydroxide resulted in a large pore volume and specific surface area in the samples. This is attributable to the optimized pore structure of the metal-containing polyacrylonitrile-based electrospun CNFs, which may provide better sites for hydrogen adsorption than do current adsorbates.
\end{abstract}

Key words: carbon nanofibers, hydrogen storage, porosity, metal catalysis, electrospinning

\section{Introduction}

Carbon nanostructures are currently among the best options for hydrogen storage due to their high surface area, low density, low cost, and facile handling [1-3]. In particular, carbon nanomaterials, such as carbon nanotubes (CNTs), fullerenes, and carbon nanofibers (CNFs), have attracted much attention because of their large surface area, and they have been considered good storage materials for molecular hydrogen. In addition to carbon nanostructures, several chemical hydrides (based on the light elements lithium, sodium and magnesium) have also been explored as potential hydrogen-storage materials [4-7]. However, these materials have proven difficult to handle and possess certain disadvantages when compared to lighter carbon nanostructures [8].

CNFs have generally been prepared using techniques such as vapor growth and plasmaenhanced chemical vapor growth $[9,10]$. However, these methods of synthesizing CNFs involve complicated and expensive processes. CNFs can also be produced by various thermal treatments and subsequent electrospinning of organic precursors, such as polyaniline, polyethylene oxide, polycaprolactone, polyacrylonitrile and similar polymer fibers. Electrospinning is an easy and efficient fabrication technique for nano- and microscale fibers [11]. Many studies have demonstrated that nanoscale carbon fibers with sizes ranging from $100 \mathrm{~nm}$ to a few microns can be produced from organic precursors using electrospinning.

In recent years, $\mathrm{CNFs}$ with various modifications have been studied for their utility as nitrogen and hydrogen adsorbents [12]. CNFs with high specific surface area and small pore sizes have been reported to adsorb significant amounts of carbon dioxide and hydrogen $[13,14]$. They have also been reported to absorb organic alcohols [15] under various experimental 
conditions. Following previous research describing the improved properties of CNFs and their potential as a hydrogen-storage medium, researchers have introduced various metals and metal precursors into CNFs. The work presented herein focuses on CNFs that were modified with embedded $\mathrm{Ti}$ and $\mathrm{Mn}$. These metals were selected for their high dispersion, solubility, affinity for hydrogen and corresponding ease of hydrogen dissociation under ambient conditions. The characteristics of these metals are useful when they are embedded into carbon nanomaterials and may ultimately improve the hydrogen adsorption properties of CNFs. In these materials, hydrogen storage is achieved either by the dissociation of hydrogen molecules into atomic hydrogen or by facilitated hydrogen physisorption onto carbon surfaces via a spillover mechanism, as has been reported in many previous studies.

This study investigated the effects of these metals on the morphological and hydrogen-storage properties of CNFs that were activated with an alkoxide at high temperatures. Compared to the metals that are involved in composite formation or are bonded to carbon or oxygen, the effects of metals on some CNFs were evidenced by the increasing adsorption capacities of these materials.

\section{Experimental}

\subsection{Raw materials}

Polyacrylonitrile (PAN) and 99.5\% N,N-dimethylformamide (DMF) were purchased from Sigma-Aldrich and were used without further purification. For the metal catalysts, 99+\% manganese powder and titanium oxysulfate $\left(\mathrm{TiOSO}_{4}\right)$ were obtained from Sigma-Aldrich (USA). A homogenous solution of $12 \mathrm{wt} \%$ PAN in DMF was prepared by mixing the materials and stirring for 5 to $10 \mathrm{~h}$ at $70^{\circ} \mathrm{C}$. Each metal catalyst $(5 \mathrm{wt} \%$ metal) was dissolved in DMF for 3 hours at $50^{\circ} \mathrm{C}$ with constant stirring. The prepared catalyst solution was slowly added to the PAN solution with continuous stirring to obtain a homogenous solution.

\subsection{Preparation of electrospun CNFs}

The CNFs were prepared using electrospinning with a sophisticated current controller that was capable of producing $60 \mathrm{kV}$; the electrospun fiber collector was held at a fixed distance from the tip (ranging from 10-15 cm). The tip and collector faced one another. The current passing through the circuit generates an electronic field, which produces small fibers that emanate from the tip of a syringe containing the polymer solution.

The metal/PAN-based fibers (in which the metals are Mn and Ti) were prepared by electrospinning a polymer/metal precursor solution containing both the metal (5 wt $\%)$ and a $12 \mathrm{wt} \%$ PAN polymer solution in DMF. The amount of the metal precursor used was limited to $5 \mathrm{wt} \%$ in all cases. The ratio of the PAN polymer and the metal content in the electrospun solution was maintained at approximately $16: 1$.

\subsection{Heat treatment and activation of CNFs}

All of the electrospun samples underwent thermal treatment at various temperatures. To stabilize or oxidize the prepared fibers, the samples were first placed in an oven and heated in air to $280^{\circ} \mathrm{C}$ at a heating rate of $1^{\circ} \mathrm{C} \min ^{-1}$. The oxidized fibers were then carbonized, which involves thermally treating the samples by heating them to $1050^{\circ} \mathrm{C}$ at a heating rate of $10^{\circ} \mathrm{C} \mathrm{min}-1$ in an inert atmosphere of nitrogen. The carbonization step is very important for obtaining a carbon mass from the precursors used in the fiber preparation. Carbonization was performed in an automated furnace equipped with a steel pipe that was placed in the center of the furnace chamber. The pipe was open at both ends and was used as the inlet and outlet for the nitrogen gas. The sample was kept in a ceramic boat and placed into the steel pipe, both ends of which were protected by the inlet and outlet flows of nitrogen. The sample was held at $1050^{\circ} \mathrm{C}$ for $1 \mathrm{~h}$, and the completion of the heat treatment required several hours to one day.

\subsection{Chemical activation using potassium hy- droxide}

A potassium hydroxide $(\mathrm{KOH})$ solution $(8 \mathrm{M}, 200 \mathrm{~mL})$ was used as the chemical activation agent. The samples (2-3 g) of the CNFs and the CNFs with the embedded metal (CNF-M; M = $\mathrm{Ti}$ or $\mathrm{Mn}$ ) were immersed in the $\mathrm{KOH}$ solution. To immerse the samples uniformly, a shaker apparatus (SK-300; JEIO TECH, Korea) was used at $70 \mathrm{rpm}$ for $10 \mathrm{~h}$. The wet CNFs and CNF-Ms were placed in an alumina boat in a steel pipe during chemical activation, and activation was conducted at $750^{\circ} \mathrm{C}$ for $3 \mathrm{~h}$ in a nitrogen atmosphere [16]. The heating rate was $5^{\circ} \mathrm{C} \mathrm{min}^{-1}$; the nitrogen feed rate was $40 \mathrm{~mL} / \mathrm{min}$. Next, the activated electrospun CNFs were washed with distilled water several times to ensure that all of the potassium was removed, which was verified by analyzing the $\mathrm{pH}$ of the filtrate after each washing. These samples were then dried at $100-110^{\circ} \mathrm{C}$ overnight.

\subsection{Characterization}

The surface morphology of the prepared samples was investigated using field-emission scanning electron microscopy (FESEM, Hitachi S-5500; Hitachi, Japan). The samples were examined without any of the coatings that are usually used in FE-SEM measurements. X-ray photoelectron spectroscopy (XPS) spectra of the samples were obtained using a MultiLab 2000 spectrometer (Thermo Electron Corporation, UK) to determine the states of the elements that were present in the samples. To study the textural properties of the samples, Brunauer-Emmett-Teller (BET) measurements were performed using a Micromeritics ASAP 2020 instrument (USA). The measurements were performed at $77 \mathrm{~K}$, with nitrogen as the adsorbent gas. To investigate the hydrogen adsorption isotherms of the prepared samples at $303 \mathrm{~K}$ and $9 \mathrm{MPa}$ of hydrogen pressure, a pressure-composition-temperature apparatus (MiraeSI Co., Korea) was used.

\section{Results and Discussion}

\subsection{Structure and morphology of CNF}

Fig. 1 presents FE-SEM images of the raw CNF, CNF-Ti and CNF-Mn indicated as (a, b), (c, d), and (e, f), respectively. CNFs with diameters as small as 200-500 nanometers were observed (Fig. 1a), and the maximum diameter of the nanofibers within 


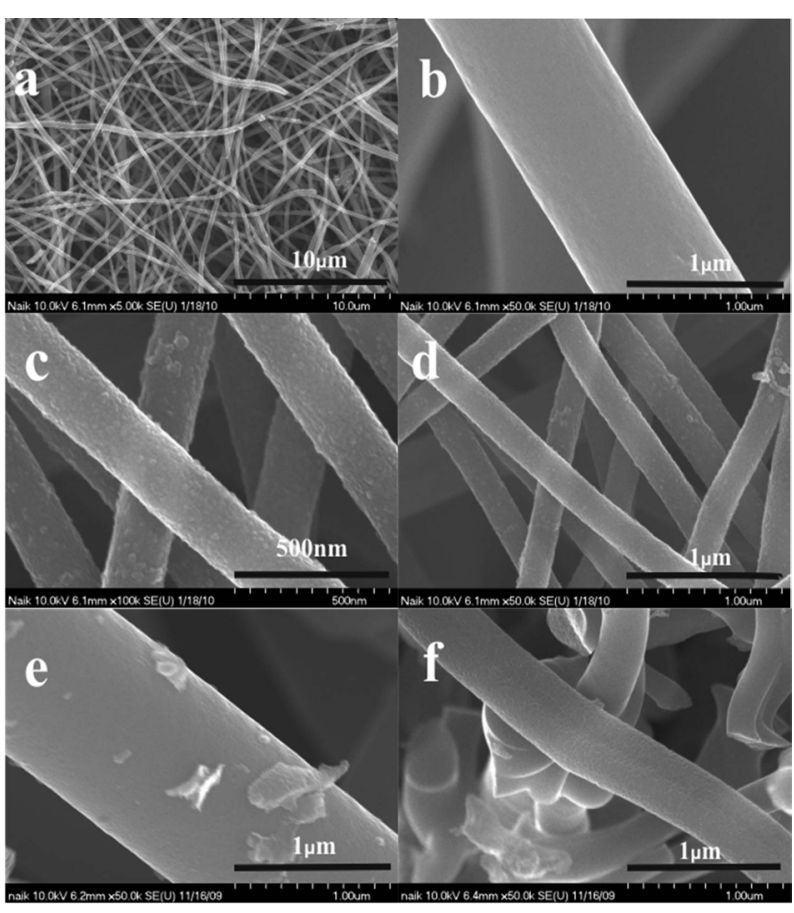

Fig. 1. Field emission-scanning electron microscopy images of activated carbon nanofibers (CNFs) with or without metal additives: $(a, b) C N F,(c, d)$ CNF-Ti, and (e, f) CNF-Mn.

the measured area was approximately 1 micron (Fig. 1b). CNFs with a smaller diameter have a higher specific surface area than larger fibers [17]; this has been reported as an important factor for achieving improved hydrogen storage capacity in carbon nanomaterials $[13,14,18]$. Metal insertion into the CNTs can lead to the degradation of the carbon surface or wall via catalytic effects at high temperatures [19]. In the case of the CNFs treated with $\mathrm{Ti}$, surface imaging after the treatment showed that the titanium traces were uniformly distributed along the CNF surfaces, with no aggregation. The particle size in the metal layer on the CNFs was observed to be approximately 10-30 nm (Fig. 1c). When using titanium oxysulfate as a dispersant, a good distribution of Ti particles was observed, which was likely due to the high solubility and hydrophilicity of the titanium precursor, which enables the efficient distribution of the Ti particles.

Additionally, other metallic precursors were selected for CNF modification due to their high solubility or dispersion and good affinity for hydrogen. These materials were also observed by FE-SEM, as shown in Fig. 1 for Mn (e and f). The Mn metal particles on the CNFs were observed to be approximately 20-40 $\mathrm{nm}$ in diameter, and some of the particles formed aggregates that were larger than $100 \mathrm{~nm}$, the Mn-only occupied regions on the CNF surfaces that were more favorable for Mn particle adsorption (Fig. 1e). In addition, some Mn particle aggregation was observed. This aggregation may be due to the formation of oxides on the CNF surfaces [20].

\subsection{Elemental analysis}

XPS is an effective tool for determining the chemical states of the modified metal atoms on the CNFs. XPS can reveal changes

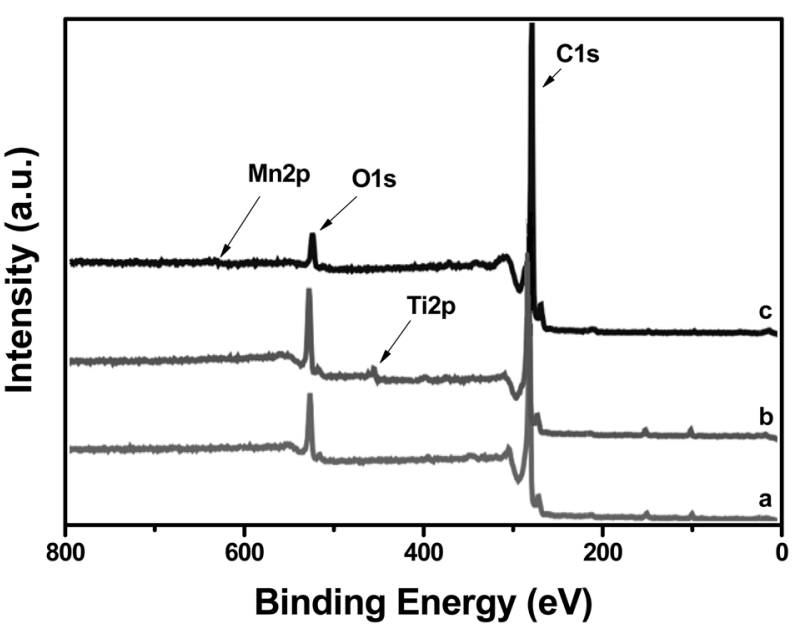

Fig. 2. X-ray photoelectron spectroscopy spectra of (a) CNFs, (b) CNF-Ti, and (c) CNF-Mn. CNF: carbon nanofiber.

in the carbon or oxygen binding energies caused by metal modification, allowing the deduction of the incorporation scheme of the metal atoms. A core-level analysis of all of the samples was performed using XPS; the results are shown in Fig. 2. The XPS spectrum of the raw CNFs exhibited two major states, $\mathrm{C} 1 \mathrm{~s}$ and $\mathrm{O} 1 \mathrm{~s}$, as indicated in trace ' $a$ ' of Fig. 2. However, the other CNFs exhibited Ti2p and Mn2p states (Figs. 2b and c, respectively), in addition to the $\mathrm{C} 1 \mathrm{~s}$ and $\mathrm{O} 1 \mathrm{~s}$ states. The binding energy of the $\mathrm{C} 1 \mathrm{~s}$ peak of the CNFs was $284.5 \mathrm{eV}$ and was not altered by titanium modification. The O1s peak of the CNF-Ti material was broad and had a binding energy of $532.0 \mathrm{eV}$. This result denotes the adsorption of Ti by hydroxyl groups [21] that are believed to result from the reaction of carbon with atmospheric moisture. The binding energy of O1s reveals that there was negligible or nonexistent Ti-O bonding in the CNF-Ti material, but the binding energy of the oxygen atoms bonded to the metal atoms is $530.5 \mathrm{eV}$ [22]. Additionally, the lowenergy side of the O1s peak increased after manganese insertion (Fig. 2c); this suggests that a fraction of the oxygen atoms was bonded to the manganese atoms in the $\mathrm{Mn}-\mathrm{O}$ bonds.

\subsection{Textural property analysis}

Fig. 3 presents the adsorption/desorption isotherms of $\mathrm{N}_{2}$ at $77 \mathrm{~K}$ for the CNF, CNF-Ti, and CNF-Mn materials. All of the isotherms followed Type-IV classification curves, which are typical of porous adsorbents that possess pores with diameters in the range of approximately 15-1000 angstroms. The slope increase at elevated pressures indicates an increased uptake of nitrogen as the pores were filled. The knee of the Type-IV isotherm generally occurs near the completion of the first monolayer. Adsorption onto the CNF-Ti material was complete at a low relative pressure, $\left(\mathrm{P} / \mathrm{P}_{0}<0.03\right)$, and the isotherm exhibited a sharply inclined knee at $\mathrm{P} / \mathrm{P}_{0}<0.03$. This narrow knee indicates that the sample has a microporous size distribution, which is clearly shown in Fig. 4. It is also important to note that, in addition to micropores, all of the samples possessed mesopores (Fig. 4). These isotherms correspond to the International Union of Pure and Applied Chemistry (IUPAC) Type-IV classification, which is associated with the simultaneous presence of both 


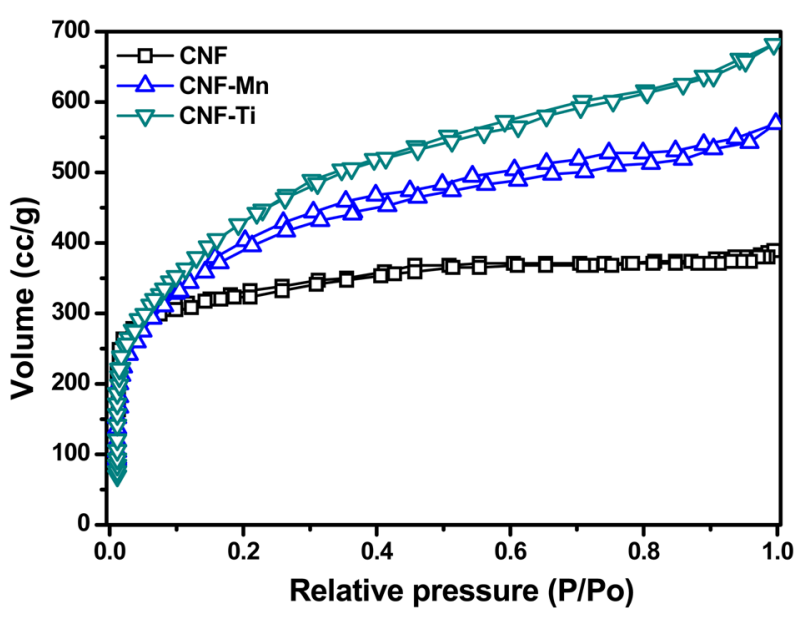

Fig. 3. Nitrogen-adsorption curves of the samples at $77 \mathrm{~K}$. CNF: carbon nanofiber.

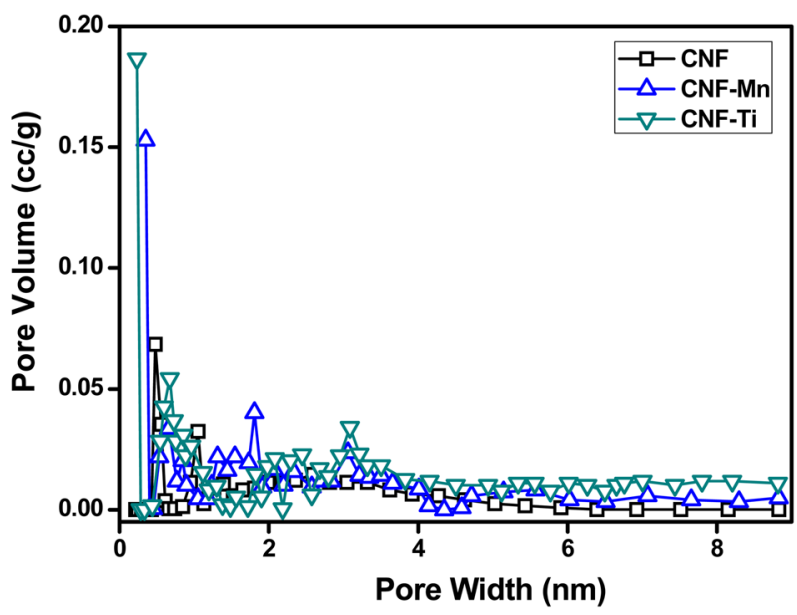

Fig. 4. Pore-size distributions of the samples. CNF: carbon nanofiber.

micropores and mesopores. There was no effect on the curve once the micropores were filled at a low relative pressure $\left(\mathrm{P} / \mathrm{P}_{0}\right.$ $<0.03$ ); however, adsorption became significant on the surfaces containing the mesopores, as indicated by the slope at a higher relative pressure [23]. During $\mathrm{N}_{2}$ adsorption/desorption, the presence of micropores is believed to provide an increased pore volume compared to that of mesopores and macropores. This pore volume corresponds to a maximum volume similar to that of the CNF-Ti sample. The narrow pore-size distribution in the
CNF-Ti material was especially related to the activation of the sample at high temperatures using $\mathrm{KOH}$ as activating agent and highly dispersed titanium metal on the electrospun fibers [24]. Comparing the isotherms of the CNF-Ti and CNF-Mn materials to those of the CNF materials revealed that the first two were superior with respect to the pore properties listed in Table 1. The knee usually occurs near the completion of the first adsorbed monolayer, and, with increasing pressure, the second and higher layers are completed until saturation is reached as the number of adsorbed layers becomes infinite. Similar results were observed in the pore-size distribution measurements, indicating the completion of adsorption in the pores. This pore-size distribution was analyzed using the Howard-Kawazoe method, with results as illustrated in Fig. 4, which reveals the clear differences in the pore characteristics of the CNF-Ti, CNF-Mn, and CNF samples.

\subsection{Hydrogen storage capacity}

Fig. 5 presents the hydrogen adsorption isotherms of the prepared samples. As expected, the maximum hydrogen storage capacity was observed in the CNF-Ti sample, followed by the CNF-Mn, and CNF samples, in that order. The CNF-Ti and CNFMn samples may have higher storage capacities because these materials have higher specific surface areas and larger micropore volumes than do the CNF materials (Table 1). The free metals in the CNF-Ti and CNF-Mn samples can also facilitate hydrogen dissociation onto the CNF surface via a spillover mechanism or can drive the hydrogen molecules into the pores [25]. The observed hydrogen storage capacities of the CNF-Ti and CNF-Mn samples were approximately $1.6 \mathrm{wt} \%$ and $1.1 \mathrm{wt}$. \%, respectively. For the raw CNF samples, the storage capacity was $0.6 \mathrm{wt} \%$ under similar conditions. The storage capacity of the CNF-Ti sample was much greater than that of the raw CNF sample, both with or without activation; this result is similar to what our group previously reported [26]. The BET results suggest that the CNF-Ti and CNF-Mn samples had greater micropore volumes and higher specific surface areas. This observation corresponds to the enhanced hydrogen storage capacities of these materials. The micropore volume of the CNF samples was much lower than those of the CNF-Ti and CNF-Mn samples, reflecting the fact that the CNF sample only adsorbed a small amount of hydrogen under similar conditions. Panella et al. [27] reported that the hydrogen storage capacities of carbon materials are proportional to their micropore volumes. However, it has previously been reported $[28,29]$ that specific surface areas and micropore volumes are not necessarily the primary markers for greater hydrogen storage capacity. In addition, it is possible that the investigated metals play intermedi-

Table 1. Comparative pore properties of the prepared samples

\begin{tabular}{ccccccc} 
Sample & $\begin{array}{c}\text { Surface area } \\
\left(\mathrm{m}^{2} / \mathrm{g}\right)\end{array}$ & $\begin{array}{c}\text { Pore volume } \\
(\mathrm{cc} / \mathrm{g})\end{array}$ & Pore width $(\mathrm{nm})$ & $\begin{array}{c}\text { Micropore } \\
\text { volume }(\mathrm{cc} / \mathrm{g})\end{array}$ & $\begin{array}{c}\text { Total pore volume } \\
(\mathrm{cc} / \mathrm{g})\end{array}$ & $\begin{array}{c}\text { Average pore } \\
\text { diameter }(\mathrm{nm})\end{array}$ \\
\hline $\mathrm{CNF}$ & 1038 & 0.490 & 1.36 & 0.10 & 0.36 & 3.13 \\
CNF-Mn & 1452 & 0.780 & 1.27 & 0.32 & 0.87 & 2.41 \\
CNF-Ti & 1574 & 0.854 & 1.27 & 0.40 & 1.04 & 2.64 \\
\hline
\end{tabular}

CNF: carbon nanofiber.

a Volume up to a 2-nm pore width. 


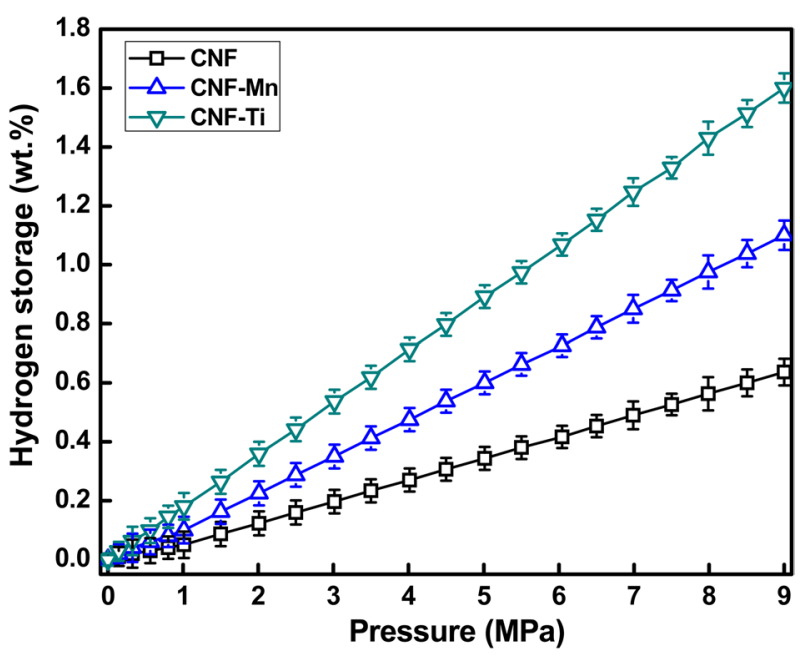

Fig. 5. Hydrogen adsorption profiles of the metal-containing carbon nanofibers at $303 \mathrm{~K}$. CNF: carbon nanofiber.

ate roles in hydrogen adsorption, perhaps by dissociating the hydrogen on the nanostructure surface or by directly forming stable metal hydrides under the studied conditions. The characteristics that drive hydrogen molecules to dissociate or spill over onto the surfaces of CNFs or that drive them into micropores and, thus, enhance the total hydrogen adsorption capacity should be considered and studied in detail in future adsorption experiments.

\section{Conclusions}

The metal catalysts $\mathrm{Ti}$ and $\mathrm{Mn}$ were homogeneously distributed along CNFs via electrospinning. A BET analysis of the prepared CNF samples showed improved surface morphology and porosity after $\mathrm{KOH}$ activation. The best catalysts for improving the surface morphology and hydrogen storage capacity were $\mathrm{Ti}$ and $\mathrm{Mn}$, which yielded CNFs with hydrogen storage capacities of approximately 1.6 and $1.1 \mathrm{wt} \%$, respectively, at $303 \mathrm{~K}$ and 9 $\mathrm{MPa}$. These results were supported by the BET analysis of the CNF-Ti and CNF-Mn materials, which had specific surface areas of 1572 and $1452 \mathrm{~m}^{2} \mathrm{~g}^{-1}$, respectively. Additionally, these materials exhibited improved micropore volumes and pore properties than did the raw CNFs (with specific surface area of $1038 \mathrm{~m}^{2} \mathrm{~g}^{-1}$ ). The results suggest that the Ti and Mn precursors were free and uniformly dispersed along the surfaces of the CNFs and did not form composites or bonds with carbon. The effect of the surface area and the porosity of the metal-inserted CNFs was evident after determining the hydrogen storage capacities and analyzing the BET results. This interpretation was also supported by the results of the XPS analyses, which were performed to better understand the phases involved during the formation of these materials.

\section{Acknowledgements}

This work was supported by grants from the National Research Foundation of Korea and the Korean Government MEST Basic Research Promotion Fund (NRF-2011-013-D00031).

\section{References}

[1] Dillon AC, Jones KM, Bekkedahl TA, Kiang CH, Bethune DS, Heben MJ. Storage of hydrogen in single-walled carbon nanotubes. Nature, 386, 377 (1997). http://dx.doi.org/10.1038/386377a0.

[2] Chen P, Wu X, Lin J, Tan KL. High H2 uptake by alkali-doped carbon nanotubes under ambient pressure and moderate temperatures. Science, 285, 91 (1999). http://dx.doi.org/10.1126/science.285.5424.91

[3] Orimo S, Majer G, Fukunaga T, Zutel A, Schlapbach L, Fujii H. Hydrogen in the mechanically prepared nanostructured graphite. Appl Phys Lett, 75, 3093 (1999). http://dx.doi.org/10.1063/1.125241.

[4] Grochala W, Edwards PP. Thermal decomposition of the non-interstitial hydrides for the storage and production of hydrogen. Chem Rev, 104, 1283 (2004). http://dx.doi.org/10.1021/cr030691s.

[5] Naik MUD, Rather SU, Zacharia R, So CS, Hwang SW, Kim AR, Nahm KS. Comparative study of dehydrogenation of sodium aluminum hydride wet-doped with $\mathrm{ScCl} 3, \mathrm{TiCl} 3, \mathrm{VCl} 3$, and $\mathrm{MnCl} 2$. J Alloys Compd, 471, L16 (2009). http://dx.doi.org/10.1016/j.jallcom.2008.03.093.

[6] Chen P, Xiong Z, Luo J, Lin J, Tan KL. Interaction of hydrogen with metal nitrides and imides. Nature, 420, 302 (2002). http:// dx.doi.org/10.1038/nature01210.

[7] Naik MUD, Rather SU, So CS, Hwang SW, Kim AR, Nahm KS. Thermal decomposition of LiAlH4 chemically mixed with Lithium amide and transition metal chlorides. Int J Hydrogen Energy, 34, 8937 (2009). http://dx.doi.org/10.1016/j.ijhydene.2009.07.003.

[8] D.K R. Hydrogen storage: the major technological barrier to the development of hydrogen fuel cell cars. Vacuum, 80, 1084 (2006) http://dx.doi.org/10.1016/j.vacuum.2006.03.030.

[9] Rodriguez NM. A review of catalytically grown carbon nanofibers. J Mater Res, 8, 3233 (1993). http://dx.doi.org/doi:10.1557/ JMR.1993.3233.

[10] Merkulov VI, Melechko AV, Guillorn MA, Simpson ML, Lowndes $\mathrm{DH}$, Whealton JH, Raridon RJ. Controlled alignment of carbon nanofibers in a large-scale synthesis process. Appl Phys Lett, 80, 4816 (2002). http://dx.doi.org/10.1063/1.1487920.

[11] Doshi J, Reneker DH. Electrospinning process and applications of electrospun fibers. J Electrostatics, 35, 151 (1995). http://dx.doi. org/10.1016/0304-3886(95)00041-8.

[12] Jung MJ, Kim JW, Im JS, Park SJ, Lee YS. Nitrogen and hydrogen adsorption of activated carbon fibers modified by fluorination. J Ind Eng Chem, 15, 410 (2009). http://dx.doi.org/10.1016/j. jiec.2008.11.001.

[13] Rzepka M, Bauer E, Reichenauer G, Schliermann T, Bernhardt B, Bohmhammel K, Henneberg E, Knoll U, Maneck HE, Braue W. Hydrogen storage capacity of catalytically grown carbon nanofibers. J Phys Chem B, 109, 14979 (2005). http://dx.doi.org/10.1021/ jp051371a.

[14] Im JS, Kwon O, Kim YH, Park SJ, Lee YS. The effect of embedded vanadium catalyst on activated electrospun CFs for hydrogen storage. Microporous Mesoporous Mater, 115, 514 (2008). http:// dx.doi.org/10.1016/j.micromeso.2008.02.027.

[15] Park C, Engel ES, Crowe A, Gilbert TR, Rodriguez NM. Use of carbon nanofibers in the removal of organic solvents from water Langmuir, 16, 8050 (2000). http://dx.doi.org/10.1021/la9916068.

[16] Kim BJ, Lee YS, Park SJ. Preparation of platinum-decorated porous graphite nanofibers, and their hydrogen storage behaviors. $\mathrm{J}$ 
Colloid Interface Sci, 318, 530 (2008). http://dx.doi.org/10.1016/j. jcis.2007.10.018.

[17] Oh GY, Ju YW, Kim MY, Jung HR, Kim HJ, Lee WJ. Adsorption of toluene on carbon nanofibers prepared by electrospinning. Sci Total Environ, 393, 341 (2008). http://dx.doi.org/10.1016/j.scitotenv.2008.01.005.

[18] Im JS, Park SJ, Kim TJ, Kim YH, Lee YS. The study of controlling pore size on electrospun carbon nanofibers for hydrogen adsorption. J Colloid Interface Sci, 318, 42 (2008). http://dx.doi. org/10.1016/j.jcis.2007.10.024.

[19] Chuvilin A, Khlobystov AN, Obergfell D, Haluska M, Yang S, Roth S, Kaiser U. Observations of chemical reactions at the atomic scale: dynamics of metal-mediated fullerene coalescence and nanotube rupture. Angew Chem Int Ed, 49, 193 (2010). http:// dx.doi.org/10.1002/anie.200902243.

[20] Zhou JH, Sui ZJ, Zhu J, Li P, Chen D, Dai YC, Yuan WK. Characterization of surface oxygen complexes on carbon nanofibers by TPD, XPS and FT-IR. Carbon, 45, 785 (2007). http://dx.doi. org/10.1016/j.carbon.2006.11.019.

[21] Ryoo MW, Seo G. Improvement in capacitive deionization function of activated carbon cloth by titania modification. Water Res, 37, 1527 (2003). http://dx.doi.org/10.1016/S0043-1354(02)00531-6.

[22] Kumar PM, Badrinarayanan S, Sastry M. Nanocrystalline TiO2 studied by optical, FTIR and X-ray photoelectron spectroscopy: correlation to presence of surface states. Thin Solid Films, 358, 122 (2000). http://dx.doi.org/10.1016/S0040-6090(99)00722-1.
[23] Jimenez V, Diaz JA, Sanchez P, Valverde JL, Romero A. Influence of the activation conditions on the porosity development of herringbone carbon nanofibers. Chem Eng J, 155, 931 (2009). http:// dx.doi.org/10.1016/j.cej.2009.09.035.

[24] Im JS, Park SJ, Kim T, Lee YS. Hydrogen storage evaluation based on investigations of the catalytic properties of metal $/$ metal oxides in electrospun carbon fibers. Int J Hydrogen Energy, 34, 3382 (2009). http://dx.doi.org/10.1016/j.ijhydene.2009.02.047.

[25] Rather SU, Zacharia R, Hwang SW, Naik MU, Nahm KS. Hyperstoichiometric hydrogen storage in monodispersed palladium nanoparticles. Chem Phys Lett, 438, 78 (2007). http://dx.doi.org/ 10.1016/j.cplett.2007.02.069.

[26] Im JS, Park SJ, Lee YS. Superior prospect of chemically activated electrospun carbon fibers for hydrogen storage. Mater Res Bull, 44, 1871 (2009). http://dx.doi.org/10.1016/j.materresbull.2009.05.010.

[27] Panella B, Hirscher M, Roth S. Hydrogen adsorption in different carbon nanostructures. Carbon, 43, 2209 (2005). http://dx.doi.org/ 10.1016/j.carbon.2005.03.037.

[28] Browning DJ, Gerrard ML, Lakeman JB, Mellor IM, Mortimer RJ, Turpin MC. Studies into the storage of hydrogen in carbon nanofibers: proposal of a possible reaction mechanism. Nano Lett, 2, 201 (2002). http://dx.doi.org/10.1021/n1015576g.

[29] Lueking AD, Pan L, Narayanan DL, Clifford CEB. Effect of expanded graphite lattice in exfoliated graphite nanofibers on hydrogen storage. J Phys Chem B, 109, 12710 (2005). http://dx.doi.org/ 10.1021/jp0512199. 